\title{
On oscillations in the additive divisor problem, 1
}

\author{
by \\ Bogdan SzydŁo (Poznań)
}

1. Introduction. In its simplest form, the additive divisor problem is to determine the asymptotic behaviour of the sum

$$
S_{k}(x)=\sum_{n \leq x} d(n) d(n+k) \quad(x>0),
$$

where $d(n)$ stands for the number of positive divisors of $n$ and $k$ is a positive integer.

On the assumption that the shift $k$ is fixed, the best result was obtained by J.-M. Deshouillers and H. Iwaniec [3], who proved that for every $\varepsilon>0$ we have

$$
S_{k}(x)=x P_{k}(\log x)+E_{k}(x)
$$

with

$$
E_{k}(x) \ll_{k, \varepsilon} x^{2 / 3+\varepsilon} \quad(x \rightarrow \infty),
$$

where $P_{k}$ is a quadratic polynomial.

On the other hand, confirming a conjecture by A. Ivić, Y. Motohashi [10] has recently proved that for each fixed $k$ we have

$$
E_{k}(x)=\Omega\left(x^{1 / 2}\right) \quad(x \rightarrow \infty) .
$$

In this note we shall prove a slight improvement of this result.

Theorem. For fixed $k \geq 1$, we have

$$
E_{k}(x)=\Omega_{ \pm}\left(x^{1 / 2}\right) \quad(x \rightarrow \infty) .
$$

The proof of (1.2) [10] (and of (1.1) [3]) proceeds via Kloosterman sums and Kuznetsov's trace formulas (cf. [2], [7], [8] and [12]). But it is perhaps easy to conceive that there should be a more direct approach avoiding these

Supported in part by KBN (grant no. 2108691 01). The article was prepared while the author was a Japanese Government (Monbusho) scholarship grantee at Tsukuba University. 
tools, namely the one using the zeta-function of our problem,

$$
\zeta_{k}(s):=\sum_{n=1}^{\infty} \frac{d(n) d(n+k)}{n^{s}} \quad(\operatorname{Re} s>1) .
$$

Moreover, within this approach it would then be natural to try to apply a certain general result of Landau [9] (cf. Lemma 0 in Section 2). Actually, we choose this line of argument.

The function $\zeta_{k}(s)$ was analyzed earlier by L. A. Takhtajan and A. I. Vinogradov [14]; see also [5] for some revision of [14]. They applied the spectral theory of the hyperbolic Laplacian (cf. [6]) directly to a modification of the Eisenstein series.

Needed facts from [14] (and [5]) will be given below in Lemmas 1 and 2 (Section 4). Lemma 3 in Section 4 (non-vanishing lemma) is not new. It is stated in [10] as a fact needed for completing the proof of (1.2). It is also remarked there that this fact is a consequence of a lemma of [11] which in turn is proved via Kloosterman sums and Kuznetsov's trace formulas. We shall prove Lemma 3 in another way.

Acknowledgements. I would like to thank Professors Matti Jutila and Yoichi Motohashi for kindly putting unpublished material at my disposal.

2. Consequence of a theorem of Landau. The following lemma is a corollary of a classical result of Landau [9] (cf. e.g. [1]).

Lemma 0. Suppose $g(x)$ is a piecewise continuous function bounded on finite intervals such that

$$
G(s):=\int_{1}^{\infty} g(x) x^{-s-1} d x
$$

converges absolutely for $\operatorname{Re} s>\sigma_{a}$. Suppose $G(s)$ analytically continues into a region including the reals $s \geq \sigma_{0}$ (with no singularity at $\sigma_{0}$ ) while $G(s)$ has a simple pole at $\sigma_{0}+i t_{0}, t_{0} \neq 0$ with residue $r$. Then

$$
\limsup _{x \rightarrow \infty} g(x) x^{-\sigma_{0}} \geq|r|, \quad \liminf _{x \rightarrow \infty} g(x) x^{-\sigma_{0}} \leq-|r| .
$$

3. Notations and auxiliary facts. The following notations will be used (cf. [5], [7] and [14]):

$$
\begin{aligned}
K_{\nu}(v):= & \int_{0}^{\infty} e^{-v \cosh t} \cosh (\nu t) d t \quad(v>0, \nu \in \mathbb{C}) \\
& (\text { the } K \text {-Bessel function); } \\
\sigma_{s}(k):= & \sum_{d \mid k} d^{s} ;
\end{aligned}
$$


$\xi(s):=\pi^{-s / 2} \Gamma(s / 2) \zeta(s)$

$d \mu(z):=y^{-2} d x d y$ (the invariant hyperbolic measure in the upper half-plane $z=x+i y, y>0)$;

$\kappa_{j}:=\sqrt{\lambda_{j}-1 / 4}$, where $\lambda_{j}$ is the $j$ th (non-zero) eigenvalue of the hyperbolic Laplacian (it is well known that $\lambda_{j}>1 / 4$ );

$z_{j}:=1 / 2+i \kappa_{j}$

$\varrho_{j}(1)$ - the first Fourier coefficient of the Maass wave form attached to $\lambda_{j}$;

$H_{j}(s):=\sum_{n=1}^{\infty} t_{j}(n) / n^{s}(\operatorname{Re} s>1)$ (the Maass $L$-function attached to $\lambda_{j}$ );

$$
E^{*}(z):=\sqrt{y}(\log y-c)+2 \sqrt{y} \sum_{\substack{n=-\infty \\ n \neq 0}}^{\infty} d(|n|) K_{0}(2 \pi|n| y) e(n x)(y>0),
$$

where $e(\alpha):=\exp (2 \pi i \alpha)$ and $c:=\log (4 \pi)-\gamma$ with Euler's constant $\gamma$;

$$
I_{k}(s):=\int_{\Pi}\left|E^{*}(z)\right|^{2} e(k z) y^{s} d \mu(z) \quad(\operatorname{Re} s>1),
$$

where $\Pi$ is the strip $|x| \leq 1 / 2, y>0$;

$$
I_{k}(w, s):=\frac{2^{2-2 s} \pi^{1-s} k^{w-s} \sigma_{1-2 w}(k) \Gamma(s-w) \Gamma(s-1+w)}{\xi(2 w) \Gamma(s)} .
$$

We will also use the following facts about the $K$-Bessel function (3.1):

$$
\begin{gathered}
K_{0}(v)>0 \quad(v>0), \\
K_{0}(v) \asymp v^{-1 / 2} e^{-v} \quad(v \rightarrow \infty), \\
\left.\left(\frac{\partial}{\partial \nu}\right)^{n} K_{\nu}(v)\right|_{\nu=1 / 2} \ll v^{-1 / 2} e^{-v} \quad(v \rightarrow \infty ; n=0,1,2), \\
\left|K_{i u}(v)\right| \leq K_{0}(v) \quad(u \geq 0, v>0), \\
K_{i u}(v) \ll e^{(-3 / 2) u} \quad(u \geq 1, v \geq 1)
\end{gathered}
$$

and

$$
\int_{0}^{\infty} K_{\nu}(t) e^{-t} t^{s-1} d t=\sqrt{\pi} 2^{-s} \frac{\Gamma(s+\nu) \Gamma(s-\nu)}{\Gamma(s+1 / 2)}
$$

$$
(\operatorname{Re}(s \pm \nu)>0) .
$$

The facts (3.5) and (3.8) follow directly from (3.1). The fact (3.6) is, of course, a corollary of the asymptotic formula for $K_{\nu}(v)$ (see [4], p. 86, (7)). The estimate (3.7) can be derived from (3.1), and (3.9) from a suitable 
integral representation for $K_{i u}(v)$ (see for example [13], (8.8)). Finally, (3.10) is a particular case of the formula (26) on p. 50 of [4].

We will use the following simple estimates from the theory of the Riemann zeta-function:

$$
\begin{gathered}
\zeta(1 / 2+i t) \ll t \quad(t \rightarrow \infty), \\
\zeta(1+i t)^{-1} \ll \log ^{7} t \quad(t \rightarrow \infty)
\end{gathered}
$$

and

$$
\int_{0}^{T}|\zeta(1 / 2+i t)|^{4} d t \ll T \log ^{4} T \quad(T \rightarrow \infty) ;
$$

see [15], (2.12.2), (3.6.3) and (7.6.1).

4. Analytic properties of $\zeta_{k}(s)$. All needed facts from [5] and [14] are stated in the following two lemmas.

LEMMA 1. The function $\zeta_{k}(s)$ can be meromorphically continued onto the whole complex plane. The only singularities of $\zeta_{k}(s)$ in the half-plane $\operatorname{Re} s \geq 1 / 2$ are: a triple pole at $s=1$ and simple poles at $s=z_{j}, \bar{z}_{j}$ $(j=1,2, \ldots)$. For $z=1 / 2+i \kappa \in\left\{z_{1}, z_{2}, \ldots\right\}$ we have

$$
\operatorname{Res}_{s=z} \zeta_{k}(s)=\frac{\sqrt{k}|\Gamma(z / 2)|^{4} \Gamma(2 \kappa i)}{(4 k)^{z} \Gamma(z / 2)^{4}} \sum_{z_{j}=z}\left|\varrho_{j}(1)\right|^{2} t_{j}(k) H_{j}^{2}(1 / 2) .
$$

Lemma 2. For $\operatorname{Re} s>1 / 2$ we have

$$
I_{k}(s)=B(s)+C(s)+D(s)
$$

where

$$
\begin{gathered}
B(s)=\left.\left(\frac{\partial}{\partial w}-c\right)^{2} I_{k}(w, s)\right|_{w=1} \\
C(s)=\frac{\pi \sqrt{k}}{(4 \pi k)^{s} \Gamma(s)} \\
\times \int_{-\infty}^{\infty} \frac{k^{-i u} \sigma_{2 i u}(k)|\xi(1 / 2+i u)|^{4} \Gamma(s-1 / 2-i u) \Gamma(s-1 / 2+i u)}{|\Gamma(1 / 2+i u)|^{2}|\zeta(1+2 i u)|^{2}} d u
\end{gathered}
$$

and

$$
\begin{aligned}
D(s)= & \frac{\sqrt{k}}{(4 \pi k)^{s} \Gamma(s)} \\
& \times \sum_{j=1}^{\infty}\left|\varrho_{j}(1)\right|^{2} t_{j}(k) H_{j}^{2}(1 / 2)\left|\Gamma\left(z_{j} / 2\right)\right|^{4} \Gamma\left(s-z_{j}\right) \Gamma\left(s-\bar{z}_{j}\right) .
\end{aligned}
$$

The above series converges absolutely. 
LEMma 3. There is a $\kappa>0$ such that

$$
\sum_{\kappa_{j}=\kappa}\left|\varrho_{j}(1)\right|^{2} t_{j}(k) H_{j}^{2}(1 / 2) \neq 0 .
$$

Pr o of. Suppose the contrary. Then by (4.2) and (4.5) we have

$$
I_{k}(s)=B(s)+C(s) \text {. }
$$

For $y>0$ consider

$$
\begin{aligned}
m(y): & =\frac{1}{2 \pi i} \int_{2-i \infty}^{2+i \infty} I_{k}(s) y^{-s} d s \\
& =\frac{1}{2 \pi i} \int_{2-i \infty}^{2+i \infty} B(s) y^{-s} d s+\frac{1}{2 \pi i} \int_{2-i \infty}^{2+i \infty} C(s) y^{-s} d s \\
& =: b(y)+c(y) .
\end{aligned}
$$

From (4.3), (3.4) and (3.10) we obtain

$$
\begin{gathered}
b(y)=\left(\frac{\partial}{\partial w}-c\right)^{2}\left[\frac{4 \pi k^{w} \sigma_{1-2 w}(k)}{\xi(2 w)}\right. \\
\left.\times \frac{1}{2 \pi i} \int_{2-i \infty}^{2+i \infty} \frac{\Gamma(s-w) \Gamma(s+w-1)}{\Gamma(s)}(4 \pi k y)^{-s} d s\right]\left.\right|_{w=1} \\
=\left.\left(\frac{\partial}{\partial w}-c\right)^{2}\left[\left(\begin{array}{c}
\text { a factor which depends } \\
\text { only on } k \text { and } w
\end{array}\right) \cdot \frac{K_{w-1 / 2}(2 \pi k y) e^{-2 \pi k y}}{\sqrt{y}}\right]\right|_{w=1} .
\end{gathered}
$$

From this and (3.7) it follows that

$$
b(y) \ll y^{-1} e^{-4 \pi k y} \quad(y \rightarrow \infty) .
$$

Next, using (4.4) and (3.10), we first obtain

$$
c(y)=\frac{e^{-2 \pi k y}}{2 \sqrt{y}} \int_{-\infty}^{\infty} \frac{k^{-i u} \sigma_{2 i u}(k)|\xi(1 / 2+i u)|^{4} K_{i u}(2 \pi k y)}{|\Gamma(1 / 2+i u)|^{2}|\zeta(1+2 i u)|^{2}} d u .
$$

Let $T \geq 2$. From Stirling's formula, (3.11)-(3.13), (3.8) and (3.9) it follows that

$$
\begin{aligned}
c(y) & \ll \frac{e^{-2 \pi k y}}{\sqrt{y}}\left[K_{0}(2 \pi k y) \int_{1}^{T}|\zeta(1 / 2+i u)|^{4} \frac{\log ^{14} u}{u} d u\right. \\
& \left.+\int_{T}^{\infty} u^{3} \log ^{14} u \cdot e^{(-3 / 2) u} d u\right] \\
& \ll \frac{e^{-2 \pi k y}}{\sqrt{y}}\left[K_{0}(2 \pi k y) \log ^{18} T+e^{-T}\right] .
\end{aligned}
$$


Putting $T:=3 \pi k y$, we obtain by (3.6),

$$
c(y) \ll y^{-2 / 3} e^{-4 \pi k y} \quad(y \rightarrow \infty) .
$$

Combining (4.7) and (4.8), we obtain

$$
m(y) \ll y^{-2 / 3} e^{-4 \pi k y} \quad(y \rightarrow \infty) .
$$

On the other hand, by (3.2), (3.3) and (4.6), we have

$$
\begin{aligned}
m(y)= & \exp (-2 \pi k y)\left[4 d(k) K_{0}(2 \pi k y)(\log y-c)\right. \\
& +4 \sum_{n=1}^{k-1} d(n) d(k-n) K_{0}(2 \pi n y) K_{0}(2 \pi(k-n) y) \\
& \left.+8 \sum_{n=1}^{\infty} d(n) d(n+k) K_{0}(2 \pi n y) K_{0}(2 \pi(n+k) y)\right] .
\end{aligned}
$$

Thus, by (3.5) and (4.9), we conclude that

$$
K_{0}(2 \pi k y) \ll y^{-2 / 3} e^{-2 \pi k y} \quad(y \rightarrow \infty) .
$$

Comparison with (3.6) gives the desired contradiction.

5. Proof of the theorem. We are going to check whether the assumptions of Lemma 0 (Section 2) will be satisfied if we put there

$$
g(x):=E_{k}(x) \quad(x \geq 1) .
$$

We have of course

$$
G(s):=\int_{1}^{\infty} g(x) x^{-s-1} d x=\frac{\zeta_{k}(s)}{s}-\sum_{\nu=1}^{3} \frac{a_{\nu}}{(s-1)^{\nu}} \quad(\operatorname{Re} s>1)
$$

with some constants $a_{\nu}(\nu=1,2,3)$. By (1.1) and Lemma 1 (Section 4) we conclude that $G(s)$ is regular in the half-plane $\operatorname{Re} s>1 / 2$ and that $G(s)$ has no singularity at $s=1 / 2$. Also, by (4.1), Lemma 3 (Section 4) and (5.1), $G(s)$ has a simple pole at some $s=z \neq 1 / 2$ with $\operatorname{Re} z=1 / 2$ such that

$$
r:=\operatorname{Res}_{s=z} G(s)=\frac{1}{z} \operatorname{Res}_{s=z} \zeta_{k}(s) \neq 0 .
$$

The theorem follows now immediately from Lemma 0 .

\section{References}

[1] R. J. Anderson and H. M. Stark, Oscillation theorems, in: Lecture Notes in Math. 899, Springer, 1981, 79-106.

[2] J.-M. Deshouillers and H. Iw aniec, Kloosterman sums and Fourier coefficients of cusp forms, Invent. Math. 70 (1982), 219-288.

[3] - - -, Additive divisor problem, J. London Math. Soc. (2) 26 (1982), 1-14. 
[4] A. Erdelyi, W. Magnus, F. Oberhettinger and F. G. Tricomi, Higher Transcendental Functions, II, McGraw-Hill, 1953.

[5] M. Jutila, The additive divisor problem and exponential sums, in: Third Conference of the Canadian Number Theory Association, Kingston 1991, Clarendon Press, Oxford, 1993, 113-135.

[6] T. Kubota, Elementary Theory of Eisenstein Series, Wiley, New York, 1973.

[7] N. V. Kuznetsov, Petersson's conjecture for cusp forms of weight zero and Linnik's conjecture. Sums of Kloosterman sums, Math. USSR-Sb. 39 (1981), 299-342.

[8] -, Convolutions of the Fourier coefficients of the Eisenstein-Maass series, Zap. Nauchn. Sem. Leningrad. Otdel. Mat. Inst. Steklov. (LOMI) 134 (1983), 43-84 (in Russian).

[9] E. Landau, Über einen Satz von Tschebyschef, Math. Ann. 61 (1905), 527-550.

[10] Y. Motohashi, The binary additive divisor problem, Ann. Sci. École Norm. Sup., to appear.

[11] —, Spectral mean values of Maass waveform L-functions, J. Number Theory 42 (1992), 258-284.

[12] N. V. Proskurin, Summation formulas for generalized Kloosterman sums, Zap. Nauchn. Sem. Leningrad. Otdel. Mat. Inst. Steklov. (LOMI) 82 (1979), 103-135 (in Russian).

[13] L. A. Takhtajan and A. I. Vinogradov, The Gauss-Hasse hypothesis on real quadratic fields with class number one, J. Reine Angew. Math. 335 (1982), 40-87.

[14] -, - , The zeta-function of the additive divisor problem and the spectral decomposition of the automorphic Laplacian, Zap. Nauchn. Sem. Leningrad. Otdel. Mat. Inst. Steklov. (LOMI) 134 (1984), 84-116 (in Russian).

[15] E. C. Titchmarsh, The Theory of the Riemann Zeta-Function, 2nd ed. revised by D. R. Heath-Brown, Clarendon Press, Oxford, 1986.

FACULTY OF MATHEMATICS AND COMPUTER SCIENCE ADAM MICKIEWICZ UNIVERSITY

MATEJKI $48 / 49$

60-769 POZNAŃ, POLAND 